\title{
Oropharyngeal Candidosis in the Older Patient
}

\author{
Kenneth Shay, DDS, MS, ${ }^{\star \dagger}$ Mary R. Trublar, DDS, $M S{ }^{\ddagger}$ and Robert P. Renner, DDS ${ }^{\ddagger}$
}

Colonization of the oral and pharyngeal regions by Candida spp., particularly C. albicans, is extremely common in humans, particularly in early and late life. A variety of local and systemic conditions predispose the transformation of the benign colonization to a pathological state, which may have severe local or serious systemic consequences. The finding of oropharyngeal candidosis in an older patient, therefore, merits investigation of the likely host factors responsible for the organism adopting its pathogenic behavior. This paper provides non-dental clinicians managing older patients a review of the clinical characteristics, risk factors, diagnosis, and management of oropharyngeal candidosis in older adults. J Am Geriatr Soc 45:863-870, 1997.

$\mathrm{N}_{\mathrm{s}}^{\mathrm{o}}$ ormally commensal organisms of the mouth, Candida sp are responsible for the most common oral mucosal lesions reported in humans, especially in early and late life. ${ }^{1}$ The lesions of oropharyngeal candidosis, usually caused by C. albicans and less commonly by C. glabrata and C. tropicalis, are reported in as many as $63 \%$ of otherwise healthy denture-wearing adults ${ }^{2}$ and more than $70 \%$ of denturewearing adults residing in hospice or long-term care facilities. ${ }^{3,4}$ In the absence of lesions, C. albicans has been identified in the saliva or within the oral cavities of $45 \%$ of healthy newborns, ${ }^{5} 45$ to $65 \%$ of healthy children, ${ }^{6}$ and 30 to $45 \%$ of healthy adults. ${ }^{3,7}$ Rates of yeast carriage are somewhat higher, 50 to $65 \%$, for patients who use removable dentures ${ }^{7}$ and are highest $(65-88 \%)$ among those of advanced age residing in acute and long-term care facilities. ${ }^{4,-11}$

Although candidosis is generally innocuous in otherwise healthy individuals, in patients with compromised immune defenses the infection can spread through the bloodstream or upper digestive system, causing mucosal ulcerations and even death. Systemic candidosis, which is caused by C. albicans 60 to $75 \%$ of the time, has been documented as a serious problem for patients receiving cytotoxic antineoplastic chemotherapy, in which it accounts for $70-80 \%$ of fungal infections. ${ }^{12}$ Candidemia in these patients carries a mortality rate of 71 to $79 \% .^{13}$

\footnotetext{
From the "Dental Service and Geriatric Research, Education, and Clinical Center, Ann Arbor VA Medical Center, Ann Arbor, Michigan; University of Michigan School of Dentistry, Ann Arbor, Michigan; and $¥$ School of Dental Medicine, State University of New York, Stony Brook, New York.

Development and publication of this manuscript was supported by PHS Grant 1D31 AH90005.

Address correspondence to Kenneth Shay, DDS, MS, Chief, Dental Service (160), VA Medical Center, 221.5 Fuller Rd., Ann Arbor, MI 48105.
}

In the oral cavity and oropharynx, Candida lesions can present with a variety of appearances, and may appear either with or without symptoms. Yet even the most circumscribed lesion represents a transformation of the normally commensal organism to a pathogenic form capable of not only a local but a disseminated effect as well. This change from commensalism to pathogen is imperfectly understood. Candida is opportunistic, transforming itself in response to changes in the complex of physical, biochemical, and biological factors that are loosely referred to as host defense. ${ }^{14}$ Because the signs and symptoms of oropharyngeal candidosis in an older patient may be closely related to serious underlying systemic problems, and because the oral disease has the potential for systemic dissemination and serious morbidity, all such lesions merit focused diagnostic and therapeutic attention.

This paper provides non-dental clinicians managing older patients a review of the clinical characteristics, risk factors, diagnosis, and treatment of oropharyngeal candidosis in older adults. Throughout we will employ the term candidosis rather than the equivalent but technically incorrect term candidiasis, inasmuch as the suffix -osis is generally used for fungal disease (e.g., "aspergillosis") whereas the suffix -iasis is generally customary for parasitic ones (e.g., "amoebiasis"). A third common term for the conditions concerning this paper, moniliasis, will also not be used here because it derives from a century-old but now corrected taxonomic error that initially placed Candida sp and Monilia $\mathrm{sp}$ (organisms found in plants and rotting wood) in the same genus. ${ }^{15}$

\section{CLINICAL PRESENTATION}

Oropharyngeal candidosis can have a number of different clinical presentations in the older individual. Oropharyngeal candidosis can be divided into acute pseudomembranous, acute atrophic, chronic hyperplastic, and chronic atrophic candidosis, and median rhomboid glossitis and angular cheilitis (Table 1). It is important to note that an oropharyngeal candidosis in a patient may present as more than just one type of lesion.

Acute pseudomembranous candidosis, often called thrush, has been well known since antiquity and represents about $30 \%$ of the clinical cases reported. ${ }^{15}$ It is most prevalent in infancy, in older people, in diabetics, patients with HIV/AIDS, leukemics, and among the terminally ill. ${ }^{16}$ It may be seen secondary to the use of steroid aerosol inhalers, ${ }^{17}$ psychotropic and other hyposalivation-inducing drugs, ${ }^{3}$ as well as in patients receiving radiation for head and neck tumors. ${ }^{18}$ It is characterized intraorally and pharyngeally by discrete white patches on the surfaces of the labial and buccal mucosa, hard and soft palate, periodontal tissues, tongue, 
Table 1. The Lesions of Oropharyngeal Candidosis

Diagnosis

Acute pseudomembranous candidosis

("thrush")

Acute atrophic candidosis

Chronic hyperplastic candidosis

("candidal leukoplakia")

Chronic atrophic candidosis

("denture stomatitis")

Median rhomboid glossitis

Angular cheilitis ("perleche")
Clinical Findings

Asymptomatic. Discrete white, raised patches that can be wiped off with gauze or tongue blade, leaving an erythematous or bleeding base. Candidal nature can be confirmed through microscopic examination and $\mathrm{KOH}$ stain, but clinical appearance is pathognomic.

Burning sensation in mouth or tongue. Tissues bright red; if tongue is involved there may be localized or generalized loss of filiform papillae.

Asymptomatic. Discrete, raised whitish lesions that range from barely palpable to hard and rough. Generally on buccal mucosa or lateral tongue; less commonly, floor of mouth, ventral tongue, or oral labial mucosa.

Asymptomatic or burning or itching; sometimes persistent salty taste. Localized or generalized erythema and edema, usually of maxillary alveolus and hard palate, limited to area covered by denture. Erythema may be pinpoint, generalized, or associated with hyperplastic, papillary nodules of the palate or palatal surface of the maxillary alveolus that bleed easily.

Often asymptomatic or associated with vague complaints of an aching tongue. Raised, whitish, depapillated rectangular area in midline and midsection of tongue.

Asymptomatic, itching, or painful; bleeds on wide opening of mouth. Erythematous fissuring at one or both corners of mouth: pale central area of serous transudate, surrounded by raised, reddened or speckled periphery. and oropharynx. The patches may coalesce to form confluent, curd-like plaques. When these plaques are rubbed off by gauze or tongue blade, often with little difficulty, the underlying base is raw, erythematous, and may bleed easily. ${ }^{15}$ The undisturbed lesion is not normally painful.

Acute atrophic candidosis is usually associated with a burning sensation in the mouth or on the tongue. The affected tissues may be bright red (Figure $1 \mathrm{~A}$ ); if the tongue is involved, there may be localized or generalized loss of the filiform papillae, and the denuded area appears distinctly glossy. The clinical appearance is indistinguishable from that arising in reaction to antibiotics or attributable to certain avitaminoses. This is a lesion in which the role of Candida infection is not fully understood: some contend that Candi$d a$ 's presence is only indicative of secondary invasion. ${ }^{15}$

Chronic hyperplastic candidosis features discrete, raised lesions that vary from small, translucent, barely palpable, whitish areas to dense opaque plaques that are hard and rough to the touch. The lesions usually occur on the buccal mucosa or lateral borders of the tongue (Figure 1B) and can only be scraped off with great difficulty. The lesions are sometimes termed Candidal leukoplakia, may be considered premalignant, and are associated with tobacco smoking. ${ }^{19}$ Yet Candida species are not always associated with oral leukoplakia, and some studies suggest the occurrence of the organism in conjunction with a premalignant, hyperplastic lesion should be regarded as a complicating factor and not a causative one. ${ }^{1,15}$

Chronic atrophic candidosis, also referred to as denture stomatitis or denture sore mouth, is regarded as the most common form of oropharyngeal infection by Candida species in older adults. Denture stomatitis is characterized by localized or generalized chronic erythema and edema of the tissues that are covered by a removable prosthesis. Lesions vary from mild hyperemic petechiae, to a generalized mild or moderate inflammatory reaction over the entire denture bearing area of the upper jaw (Figure $1 \mathrm{C}$ ), to a severe inflammatory reaction of the mucosa with the formation of highly vascularized, papillary nodules (usually in the center and anterior portions of the hard palate: Figure 1D). Lesions of denture stomatitis are usually confined to the palate and upper jaw but may affect the mandibular tissues. Lesions are usually painless but some individuals experience slight soreness. If present, hyperplastic papillary nodules may ooze blood when lightly rubbed.

Median rhomboid glossitis is a chronic, elevated, symmetrical area of hypoplasia or atrophy of the filiform papillae on the tongue dorsum anterior to the circumvallate papillae. Biopsy of median rhomboid glossitis lesions reveals candidal hyphae in more than $85 \%$ of cases. ${ }^{15}$

Angular cheilitis, also termed perleche, is an erythematous fissuring at one or both corners of the mouth. The center 


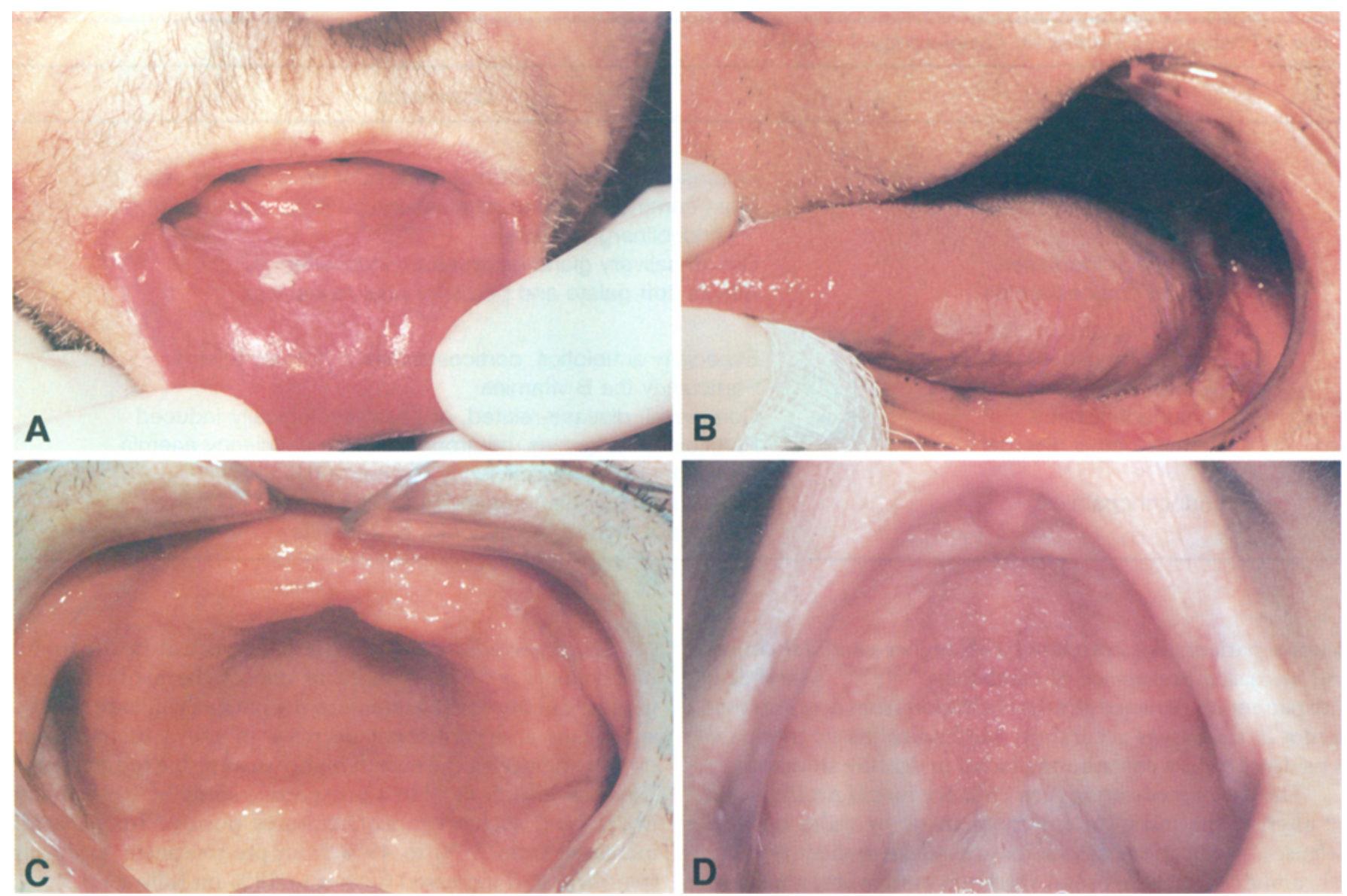

Figure 1.(a) Acute atrophic candidosis associated with cyclosporine use (photograph provided by Dr. Randy Huffines); (b) Chronic hyperplastic candidosis on dorsolateral tongue of patient with a $100+$ pack-year history of cigarette smoking. Biopsy reveals hyperkeratosis without dysplasia; (c) Chronic atrophic candidosis, mildest form; (d) Chronic atrophic candidosis, most severe form (photograph provided by T. Bloem).

of the lesion is usually white, denuded, and weeping. The periphery may be raised and reddened or speckled. The lesion can be asymptomatic, itching, or painful, and commonly bleeds when the patient opens the mouth wide. Angular cheilitis may occur in isolation, but it is usually associated with an intraoral candidal infection. In older patients, the tendency for the lesion is worsened by the chronically moist environment that comes from facial wrinkling both at the corners of the mouth and along the nasolabial fold. This is frequently worse in patients who are long-term denture wearers, as the gradual resorption of the bone on which the dentures rest reduces the height of the lower face when the mouth is closed. Cheilitis lesions are often superinfected with Staphylococcus epidermidis; ${ }^{20}$ they have also been linked to riboflavin deficiency. ${ }^{21}$

\section{RISK FACTORS}

Yeasts are normal members of the intraoral microflora in a substantial proportion of the population. Yet under certain conditions (Table 2) the opportunistic organisms become virulent in response to changes in the oral environment and/or a decreased defensive capability of the host. Oropharyngeal candidosis may come about as a result of dentures, use of certain medications, changes in the saliva, damage to the epithelial barrier, or impairment in immunity attributable to disease state or therapy. The compounding of two or more of these predisposing factors, as is often the case with a frail older patient, may further increase the probability of the individual developing an overt infection and complicate management and resolution of the condition once it occurs.

The presence of dentures - complete or partial - provides Candida with both a compatible surface on which to grow and a sheltered, favorable environment in which to thrive. Studies have consistently demonstrated greater presence of yeast among denture wearers,,${ }^{2,9,22-24}$ as well as a higher prevalence of candidosis ${ }^{2,7}$ compared with non-denturewearing, age-matched controls.

The presence of a denture, even a removable partial denture, creates a local microenvironment between the prosthesis and the underlying tissue. Flow from minor salivary glands and the free exchange of oxygen with the rest of the mouth are impeded by the presence of the denture. The resulting low $\mathrm{pH}$, anaerobic environment favors the growth of Candida and other saprophytic organisms. Candida has an affinity for the intaglio (tissue-fitting) surface of dentures and adheres to salivary proteins on the prosthesis. ${ }^{25}$ Cultures taken from patients with oropharyngeal candidal infections consistently show greater colonization of the intaglio surface by Candida than of the tissue surface itself. ${ }^{10}$ Failure to remove the denture pellicle through thorough daily hygiene practices increases the likelihood for attachment of an infectious microflora that includes Candida species. Similarly, wearing dentures throughout a 24-hour period and removing them only briefly for cleaning, as approximately $75 \%$ of 
Table 2. Predisposing Factors for Oropharyngeal Candidosis

Factors

Comments

Local factors

Denture use

Salivary hypofunction

Head/neck irradiation

Topical corticosteroids

Systemic factors

Medication use

Nutritional deficiency

Immunodeficiency

Hematological disorders

Endocrinopathies
Increased risk with poor oral care, poor fit, nocturnal use

Most commonly due to medication use (e.g., agents with anticholinergic effects)

Due to salivary gland destruction, mucositis

Affects soft palate and posterior pharyngeal wall

Especially antibiotics, corticosteroids, cytotoxic agents

Particularly the $B$ vitamins

Congenital, disease-related, or pharmacologically-induced

Particularly leukemias, neutropenias, iron deficiency anemia, myeloperoxidase deficiency of neutrophils

Most commonly diabetes mellitus; also hypofunctional states of thyroid, parathyroid, or adrenals denture patients do, correlates with higher candidal counts and increased prevalence of candidosis. ${ }^{7,22,26,27}$ Many denture patients experience physical irritation from dentures that do not fit the tissues accurately. Mechanical tissue trauma is considered to be a contributing factor in denture stomatitis. ${ }^{26}$

The use of medications can enhance fungal proliferation by altering the intraoral environment or by reducing host defenses. Medications associated with development of clinical candidosis include broad spectrum antibiotics, glucocorticosteroid preparations, and antisialogogue and immunosuppressive agents. The use of broad spectrum antibiotics may result in suppression of the normal oral bacterial flora with an overgrowth of the endogenous candidal organisms, although the data supporting this widely-accepted mechanism are largely anecdotal. ${ }^{1}$ It is currently believed that the action of antibiotics limits bacterial adherence to oral epithelium, thereby opening a broader niche for candidal colonization. ${ }^{14}$ Steroid inhaler medications appear to lower mucosal resistance to Candida by locally suppressing both the nonspecific inflammatory and cell-mediated T-lymphocyte responses. ${ }^{18}$ Systemic steroid regimens also impair cellular immunity by an analogous mechanism. ${ }^{15}$

Saliva is critical for maintaining oropharyngeal health. It maintains a nearly neutral $\mathrm{pH}$, contains the nonspecific antimicrobial factors lactoferrin, lysozyme, and lactoperoxidase, is an abundant source of secretory $\operatorname{IgA}$ and fungistatic histidine-rich polypeptide, and maintains epithelial health through epidermal growth factor and lubrication of the tissues with a variety of glycoproteins. ${ }^{12,28}$ Alteration of the quality or quantity of saliva is potentially devastating to the health of the oral cavity and protection of the individual in general and specifically lowers host resistance to candidal infection. ${ }^{14}$ Yet an extremely common side effect of medications is the modification of salivary flow and composition. ${ }^{28}$ Agents with anticholinergic effects (e.g., anticholinergics, tricyclic antidepressants, neuroleptics, antihistaminics) create the most concern. ${ }^{3} \mathrm{~A}$ number of systemic disease states are also associated with diminished salivary flow and, thereby, with the development of oropharyngeal candidosis. In older patients, these include hypothyroidism, hypoadrenalism, and Sjögrens syndrome (SS). 29
During irradiation of the head and neck as treatment for malignancy, if $50 \%$ or more of one of the major salivary glands is in the field of radiation the patient will experience a significant and permanent decrease in saliva quantity and quality, rendering the mouth highly susceptible to fungal and bacterial infections. ${ }^{30}$ In a prospective study of 109 patients undergoing such treatment, cultures positive for Candida increased from $27 \%$ to $49 \%$ from pre-treatment to during treatment and increased further to $59 \%$ on follow up. ${ }^{19}$

In patients with diabetes, fungal infections may be more severe and complicated secondary to neutrophil suppression. ${ }^{31} \mathrm{Aly}$ et al. have identified age, poor glycemic control, blood type, and the presence of dentures as factors that influence the oral carriage of yeast in the diabetic patient. ${ }^{32}$ Hill et al. ${ }^{33}$ found that glycosylated hemoglobin greater than $12 \mathrm{mg} \%$ was strongly associated with candidal infection.

An individual's blood type may play a role in host susceptibility to candidal infections by influencing the ability of the organism to adhere to epithelial cells. Several hexoses and hexosamines have been identified as receptors for Candida, and these glycocompounds are the immunodominant sugars of blood types. Denture stomatitis and plaque accumulation have been found to be statistically more severe and in greater quantity in patients of blood group $\mathrm{O}$ than the other ABO blood types in several studies. ${ }^{34}$ Tobacco smokers generally display higher candidal carriage and greater prevalence of candidosis, probably related to the decrease in oral oxygenation and decreased oral leukocyte and oral immunoglobin concentration observed in smokers. ${ }^{35}$

Immunosuppressive agents such as azathioprine selectively suppress the $\mathrm{T}$-lymphocyte system and increase host susceptibility to fungal infections. ${ }^{36}$ Most antineoplastic cytotoxic chemotherapy regimens cause a transient but serious diminution in immune system function. In one investigation, $46.9 \%$ of 1500 patients treated for acute leukemias developed chemotherapy-related oral complications, of which $34.2 \%$ were oral infections and Candida the most frequently implicated pathogen. ${ }^{37} \mathrm{Wahlin}^{38}$ suggested that some of the increase in candidosis in leukemic patients was due to salivary suppression brought on by the cytotoxic chemotherapy. Adjuvant chemotherapy for solid tumors has a less common 
but still serious effect, with $9.7 \%$ of patients contracting infections and Candida responsible for more than $70 \%$ of those infections. ${ }^{39}$

Altered immunological states such as those seen in patients with the human immunodeficiency virus render an individual extremely susceptible to colonization and the development of oropharyngeal candidosis. ${ }^{36}$ Primary immune deficiencies such as HIV, IgA deficiency, and lymphocytic and phagocytic dysfunction are typically accompanied by oropharyngeal or disseminated candidosis. It has been suggested that cellular factors are the primary mechanism of defense against oral mucocutaneous infections and that humoral factors are extensively involved in the containment and the prevention of systemic infections. ${ }^{36,40}$ Porter and Scully reported that candidosis infections were present in $25 \%$ of 39 IgA-deficient patients studied. ${ }^{41}$

Finally, diet plays a multifactorial role in the development of oral candidal infections. Growth of Candida in saliva is enhanced by the presence of glucose. The adherence of the organism to oral epithelial cells is increased by a high carbohydrate diet. ${ }^{21}$ Correlations have been reported between low serum iron and folate concentrations and the development of candidal infections, particularly angular cheilitis. ${ }^{24}$ The suggested mechanism is a decreased lymphocytic response to Candida antigen in iron deficient states; responsivity is restored upon correction of the deficiency. ${ }^{1}$ Nutritional deficiencies, in general, can result in diminished integrity of the mucosal barrier and allow for increased candidal colonization. In addition to iron and folate, Vitamins $B_{1}, B_{2}, B_{6}$, and $C$ have been implicated as contributing to candidosis ${ }^{21}$ by predisposing patients to candidal invasion of epithelium. ${ }^{42}$

\section{DIAGNOSIS}

The clinical presentation of oropharyngeal candidosis is frequently unambiguous, but laboratory testing is necessary to have a positive confirmation, to differentiate the lesion from lichen planus, benign mucous membrane pemphigoid, pemphigus, squamous cell carcinoma, or other mucosal lesions, and to rule out a possible role of vitamin deficiency. Authors have suggested sampling for culture using swabs (on the tongue, ${ }^{24}$ palate ${ }^{8,2.3}$ throat, ${ }^{24}$ or denture ${ }^{2,23}$ ), sponges (on the tissues ${ }^{7,24}$ or on the denture ${ }^{7}$ ), rinses, ${ }^{10}$ saliva, $8,22,43$ and dental impressions (of either the mouth ${ }^{9}$ or the inner surface of the denture ${ }^{9,44}$ ). But inasmuch as one-third to one-half of the population have Candida present in their oral cavities, positive cultures alone are inadequate for a diagnosis. Similarly, a negative culture or cytological smear does not definitively rule out a fungal cause, as illustrated by the widely variant findings obtained by comparisons of salivary cultures, oral rinse cultures, sponge imprints of tissue and denture, denture scrapings, and epithelial smears. ${ }^{2,7,10,15,22}$ Quantity of candidal carriage correlates with infection, so a quantitative determination is necessary to delineate benign carriage from an infected state. Epstein et al. suggest patients rinse with phosphate buffered saline for 60 seconds and expectorate. When the expectorant is centrifuged, and the concentrate pellet re-suspended and cultured on Sabouraud's media for 48 to 72 hours at $35^{\circ} \mathrm{C}$, growth $\geq 200 \mathrm{cfu} / \mathrm{mL}$ is indicative of infection rather than merely carriage. ${ }^{45}$

Histological confirmation can be achieved with $\mathrm{KOH}$, PAS, or H\&E stains, but biopsies are prone to false negative results, particularly in chronic atrophic candidosis, where far greater fungal populations are found on the denture intaglio surface than on the tissue. ${ }^{1}$ Exfoliative cytology can be accomplished easily with a tongue blade firmly scraping the surface of a lesion. The material is then transferred to a slide, fixed with ethanol, and stained. Generally the presence of hyphal (filamentous) forms of C. albicans among the epithelial cells is confirmatory for infection, but another (although less common) oral yeast, C. glabrata, does not display a hyphal form even when pathogenic. ${ }^{15}$ And the blastospore (budding) form of C. albicans, while not a tissue invader like its hyphal form, may still be responsible for disease through noninvasive mechanisms (such as tissue reaction to metabolites). ${ }^{40}$

In general, the clinical appearance of acute pseudomembranous and chronic atrophic candidosis will be sufficient for diagnosis and initiation of treatment (described below). Culture and sensitivity testing should be undertaken if initial therapy is unsuccessful. In contrast, acute atrophic and chronic hyperplastic forms may mimic other mucosal lesions, or those lesions may be complicated by candidal superinfection. Biopsy is recommended, even as empiric therapy is initiated, to rule out multifactorial or more serious disease.

\section{MANAGEMENT}

Management of the patient with oral candidosis requires the identification and, if possible, concurrent resolution of underlying predisposing systemic conditions. In addition, the clinician must assess current medication use (to identify agents that either predispose the patient to the infection or might interact with the antifungal regimen), concurrent mucosal disorders (that may be treated with systemic steroids), use of oral prostheses, and the type, severity, and chronicity of the infection.

Cases of oral candidosis that appear not to be complicated by systemic factors are generally managed through improvement of oral hygiene and the use of topical antifungal rinses on a schedule of four times a day for 2 weeks. Good oral hygiene consists of the care and cleaning of the dentition, soft tissue, tongue, and - most importantly - dentures, if present. If a patient wears dentures, dental referral is advisable because modification of the dentures' fit and function may be required. In any case, patients should be instructed to leave their dentures out overnight or for one extended period daily (at least 6 hours). The patient must be reminded to remove the denture while rinsing with the antifungal agent to permit contact between the medication and the mucosa.

It is essential that the denture be cleaned and disinfected on a daily basis to prevent reinfection. Dentures have irregular and porous surfaces to which Candida readily adheres and in which it can reside, ${ }^{46}$ protected from mechanical debridement such as brushing. A comparison of fungal contamination of dentures cleaned by brushing and those soaked in a commercial enzyme-containing denture cleaning solution found that soaking alone was as effective in eliminating Candida as soaking and brushing and far more effective than brushing without soaking. ${ }^{47}$ Soaking the denture in a $0.12 \%$ chlorhexidine solution has been demonstrated to be effective $^{48}$ although soaking in nystatin solution is not. ${ }^{49}$ Disinfection can also be accomplished by soaking the denture in a 1:10 dilution of household bleach (although this approach should not be recommended for a metal-containing denture, as discoloration will result), commercial denture cleanser (alkaline peroxide), ${ }^{50}$ or benzoic acid. ${ }^{51}$ Use of an ultrasonic cleaning tank with a suitable solution was shown by scanning 
electron micrography to be more effective in removing debris and organisms than soaking alone. ${ }^{52}$ Air drying the denture also kills adherent yeast. ${ }^{53}$

Therapeutic agents whose indications and usage include the treatment of oral candidosis are listed in Table 3. The most commonly prescribed topical rinse is nystatin, ${ }^{54}$ which is absorbed only sparingly through the GI tract, skin, and mucous membranes. Although repeated in vitro subculturing of C. albicans exposed to nystatin has been shown to result in

Table 3. Summary of Management Strategies for Oropharyngeal Candidosis

\section{Denture care}

Clean at least daily with running water and a brush specifically for cleaning dentures

After brushing, soak daily for 20 minutes in a fresh

solution of one of the following:

$0.12 \%$ chlorhexidine gluconate

commercial denture cleanser (e.g., Polident $B$,

Efferdent\&)

1:10 solution of household bleach:water

benzoic acid solution

Alternatively, place denture in one of the above solutions and run in ultrasonic cleaner 20 minutes

Keep dentures out of mouth for at least 6 hours per day, preferably longer

Allowing a brushed-clean denture to dry in air will also eradicate adherent fungi

Have prostheses evaluated for proper tissue adaptation and occlusion by a dentist

Care of intraoral mucosa

Topical agents (For all these agents, remove dentures

before use of medicament, then thoroughly clean

dentures before reinserting)

Nystatin suspension (commercially prepared)

$100,000 \mathrm{U} / \mathrm{mL}$ in sucrose solution $5 \mathrm{~mL}$ QID, rinse $60 \mathrm{sec}$ and spit or swallow.

Nystatin suspension (extemporaneously prepared) $100,000 \mathrm{U} / \mathrm{mL}$ (no sucrose) $5 \mathrm{~mL}$ QID, rinse $60 \mathrm{sec}$ and spit or swallow.

Nystatin oral troches. Suck until dissolved, TID/QID

Nystatin vaginal suppositories. Suck until dissolved, TID/QID

Chlorhexidine gluconate $0.12 \%, 15 \mathrm{~mL}$ BID rinse 60 sec and spit

Clotrimazole troches $10 \mathrm{mg}$. Suck until dissolved, 4-5 $\times /$ day

Clotrimazole vaginal suppositories, suck until dissolved, $1-3 \times /$ day

Systemic agents

Fluconazole tablets (100 mg first day; $50 \mathrm{mg} \mathrm{QD} \mathrm{for}$ 10 days)

Ketoconazole tablets (200 mg TID for 10 days)

Care of lip commissures ("angular cheilitis")

Nystatin ointment 100,000 U/g apply TID/QID

Clotrimazole 1\% cream apply TID/QID

Clotrimazole 1\% ointment apply TID/QID

Ketoconazole 2\% cream apply TID/QID

Nystatin/diphenhyḍramine ointment apply TID/QID

Nystatin/triamcinolone acetonide ointment apply TID/QID the development of nystatin-resistant isolates, ${ }^{55}$ other studies found no clinically significant emergence of resistance even in severely susceptible patients. ${ }^{56}$ Prepared nystatin oral rinses customarily contain more than $50 \%$ sucrose to disguise a disagreeable taste. This can promote tooth decay in older patients with teeth, particularly those who suffer from salivary hypofunction, but a sugar-free nystatin rinse can be prepared by a pharmacist. Another option is for the dentate patient to suck on nystatin or clotrimazole vaginal suppositories, and a third option is to use one of the systemic agents described below.

Nystatin is relatively ineffective if the candidosis is complicated by diabetes, steroid use, or an immunocompromised or immunosuppressed state. Clotrimazole troches are effective for some cases that do not resolve with the use of nystatin ${ }^{57}$ but share with nystatin the need for repeated dosages throughout the day and the disadvantage of high sugar content. For these reasons, the single dose per day of fluconazole has gained popularity in recent years, and itraconazole, also prescribed for once-per-day dosing, shows promise as well. ${ }^{58}$ Ketoconazole is also highly effective, although drug interactions (see Table 4), hepatic effects, and the need for gastric acidity for proper absorption make it unsuitable for many geriatric cases. It has also been suggested that ketoconazole may be ineffective in patients with salivary hypofunction because the agent is secreted in the saliva. ${ }^{41}$

Clinical relapse following the chemotherapeutic management of chronic atrophic candidosis is not uncommon. In many cases, this is probably caused by poor patient compliance with some aspect of the treatment regimen. ${ }^{56}$ Compliance can be compromised by: patients not experiencing discomfort and, therefore, not perceiving the need for the medication; the expense or disagreeable taste of the medication; failure to remove the dentures when using the medication; inadequately improved denture hygien ${ }^{59}$; or failing to remove the prostheses for at least 6 hours each day. In the institutional setting, compliance may be compromised further by decreased patient cooperation, inability of the patient to retain the antifungal agent in the mouth, and lack of staff awareness of the necessity to remove and clean the denture

Table 4. Potential Drug Interactions with Systemic Antifungals

Interaction

Ketoconazole Fluconazole

Decreased circulating azole levels

Antacids

$\mathrm{H}_{2}$ Blockers

Isoniazid

Rifampin

Increased circulating levels of

Warfarin

Cyclosporin

Oral hypoglycemics

Corticosteroids

Decreased circulating levels of Theophylline

Decreased levels of azole and Phenytoin

Cardlovascular reaction

Terfenadine

$\begin{array}{ll}+ & - \\ + & - \\ + & + \\ + & + \\ + & + \\ + & + \\ + & + \\ + & + \\ + & + \\ + & + \\ + & +\end{array}$


for each medication administration. If noncompliance is suspected, the use of single dose per day systemic regimens and assistance with hygiene may be advisable. The concept that patients may reinfect themselves from other, non-oral body sites that are acting as candida reservoirs (e.g., nasolabial or inframammary folds) also has led some authors to advocate the use of systemic regimens over local therapy in recalcitrant cases. ${ }^{15}$

Antifungal steroid creams and ointments are useful for the treatment of angular cheilitis although any concurrent intraoral infection must be managed if the perioral lesion is to be resolved. Furthermore, the contribution of dietary deficiency should always be explored for findings of this lesion, even as topical treatment is initiated. The patient should apply the prescribed cream or ointment (nystatin, clotrimazole, ketoconazole, or miconazole) to the infected area at least twice a day for 2 weeks. Empirically, these preparations have been suggested for cases of chronic atrophic candidosis, where the cream is placed on the intaglio surface of the denture.

Chlorhexidine gluconate oral rinse can be effective in uncomplicated cases of oropharyngeal candidosis when appropriate attention is paid to denture hygiene. ${ }^{48}$ It can also be an appropriate adjunctive therapy to systemic antimycotic regimens. Kulak et al. found that fluconazole in conjunction with chlorhexidine gluconate for the treatment of simple denture stomatitis was better than management with fluconazole alone or the remaking of the denture without medication. ${ }^{60}$ Chlorhexidine should not be used in combination with nystatin rinse, however, as a reduction in the efficacy of both medications results. ${ }^{61}$

The potential morbidity of oropharyngeal candidosis in certain groups of immunosuppressed patients poses the possibility for prophylaxis against fungal infection in these individuals. Nystatin rinses have not been successful; despite prophylactic nystatin rinsing during conventional chemotherapy and bone marrow transplantation, about one-third of leukemic patients develop oropharyngeal candidosis. ${ }^{62}$ Neither nystatin rinse nor clotrimazole troches were effective in eliminating all incidences of candidosis in a series of immunosuppressed liver transplant patients. ${ }^{63}$ In contrast, prophylactic use of chlorhexidine rinse has been quite effective. Ferretti et al. reported highly significant success with bone marrow transplant patients, in which cultivable Candida was reduced and no infections were noted clinically when patients rinsed with chlorhexidine; nearly $70 \%$ of the control group developed oropharyngeal candidosis, and two died of systemic candidoses. ${ }^{64}$ Fluconazole was also found to be effective in a prophylactic regimen of $50 \mathrm{mg} /$ day in a randomized, double-blind trial involving 112 patients with metastatic neoplasms. ${ }^{65}$ Although equal proportions of both groups cultured positive for Candida at the outset of the study, only $3 \%$ of the fluconazole group but $54 \%$ of the controls developed acute pseudomembranous candidosis in the 4 weeks of the trial.

Prophylactic antifungal treatment is less defensible for patients who are at high risk for uncomplicated oropharyngeal candidosis but at low risk for serious local and/or systemic spread. This includes otherwise healthy denture wearers, patients with drug-induced xerostomia, diabetics, and those about to undergo irradiation of the head and neck for treatment of malignancy. For these patients, the recommended care is prevention through oral and denture hygiene and periodic oral examinations followed by definitive treatment upon the discovery of fungal infection.

New therapeutic agents as well as sustained delivery systems are under development and investigation. Incorporation of amphotericin B powder into denture adhesive was not successful, possibly because of the amounts employed. ${ }^{66} \mathrm{Al}-$ ternatively, inclusion of nystatin powder in a dentist-applied soft denture liner showed a sustained antifungal effect in vitro. ${ }^{67}$ Preliminary work on the use of a rinse containing histidine-rich polypeptide (HRP) - a natural antifungal substance in normal human saliva - revealed a reduction and/or elimination of C. albicans from denture fitting surfaces. ${ }^{68} \mathrm{~A}$ mucosal oral therapeutic system (MOTS) designed to provide a sustained 2-hour release of nystatin using a controlledrelease osmotic tablet has been found to be superior to nystatin pastilles. ${ }^{69}$ Miconazole-containing lacquer applied to the intaglio surface of a denture effectively reduces candidal colonization, but current formulations have to be reapplied frequently. ${ }^{70}$

\section{SUMMARY}

Oropharyngeal candidosis is an extremely common oral infection, particularly among older adults. Its occurrence represents a local or systemic breakdown in normal host defenses, making its appearance an alarm that should not be ignored. Clinicians caring for older adults should be familiar with the clinical presentations of oropharyngeal candidosis, its signficance as a physical finding, and the range of management options available.

\section{REFERENCES}

1. Budzt-Jørgensen E. Etiology, pathogenesis, therapy, and prophylaxis of oral yeast infections. Acta Odontol Scand 1990;48:61-69.

2. Theilade E, Budtz-Jorgensen E, Theilade J. Predominant cultiviable microflora of plaque on removable dentures in patients with healthy oral mucosa. Arch Oral Biol 1983;28:675-680.

3. Lucas VS. Association of psychotropic drugs, prevalence of denture-related stomatitis and oral candidosis. Community Dent Oral Epidemiol $1993 ; 21: 313-316$.

4. Aldred MJ, Addy M, Bagg J, Finaly 1 . Oral health in the terminally ill: A cross-sectional pilot survey. Spec Care Dentist 1991;11:59-62.

5. Manning DJ, Coughlin RP, Poskitt EM. Candida in mouth or on dummy? Arch Dis Child 1985;60:381-382.

6. Berdicevsky I, Ben-Aryeh H, Sazargel R, Gutman D. Oral Candida in children. Oral Surg Oral Med Oral Pathol 1980;57:37-40.

7. Arendorf TM, Walker DM. The prevalence and intra-oral distribution of Candida albicans in man. Arch Oral Biol 1980;25:1-10.

8. Cumming CG, Wight $C$, Blackwell CL, Wray D. Denture stomatitis in the elderly. Oral Microbiol Immunol 1990;5:82-85.

9. Cardash HS, Helft M, Shani A, Marshak B. The prevalence of Candida albicans in denture wearers in an Israeli geriatric hospital. Gerodontology 1989;4:101-107.

10. Jobbins J, Bagg J, Parsons $K$ et al. Oral carriage of yeasts, coliforms and staphylococci in patients with malignant disease. J Oral Pathol Med 1992;21:305-308.

11. Holbrook WP, Hjörleifsdóttir DV. Occurrence of oral Candida albicans and other yeast-like fungi in edentulous patients in geriatric units in Iceland. Gerodontics 1986;2:153-156.

12. Redding SW, Rinaldi MG, Hicks JL. The relationship of oral Candida tropicalis infection to systemic candidosis in a patient with leukemia. Spec Care Dentist 1988;8:111-114.

13. Meunier-Carpentier E, Williams AF, Howell A. Fungemia in the immunocompromised host. Am J Med 1981;71:363-370.

14. Epstein JB, Truelove EL, lzutzu KL. Oral candidiasis: Pathogenesis and host defense. Rev Infect Dis 1984;6:96-106.

15. Samaranayake L.P. Nutritional factors and oral candidosis. J Oral Pathol $1986 ; 15 ; 61-6.5$.

16. Fotos PG, Hellstein JW. Candida and candidosis. Dent Clin North Am 1992;36:8.57-878. 
17. Finlay IG. Oral symptoms and Candida in the terminally ill. Br Med J 1986;292:592-593.

18. Salzman GA, Pyszczynski DR. Oropharyngeal candidiasis in patients treated with beclomethasome dipropionate delivered by metered-dose inhaler alone and with Acrochamber. J Allergy Clin Immunol 1988;81:424-428.

19. Silverman S, Luangjarmekorn L, Greenspan D. Occurrence of oral Candida in irradiated head and neck cancer patients. J Oral Med 1984;39:194-196.

20. Arendorf TM, Walker DM, Kingdom RJ et al. Tobacco smoking and denture wearing in oral candidial leukoplakia. Br Dent J 1983;155:340-343.

21. Öhman SC, Jontell $M$. Treatment of angular cheilitis: The significance of microbial analysis, anti-microbial treatment, and interfering factors. Acta Odontol Scand 1988;46:267-272.

22. Arendorf TM, Walker DM. Oral candidial populations in health and disease. Br Dent J 1979;147:267-272.

23. Palmquist $S$, Unell $L$, Linquist $B$. Denture stomatitis in nursing home patients. Swed Dent J 1984;8:73-80.

24. Wilkieson C, Samaranayake LP, MacFarlane TW et al. Oral candidosis in the elderly in long-term hospital care. J Oral Pathol Med 1991;20:13-16.

25. Samaranayake LP, McCourtie J, MacFarlane TW. Factors affecting the in vitro adherence of Candida albicans to acrylic surfaces. Arch Oral Biol 1980;25:611-615.

26. Budtz-Jorgensen E, Stenderup A, Grabowski M. An epidemiologic study of yeasts in elderly denture wearers. Commun Dent Oral Epidemiol $1975 ; 3: 115-119$.

27. Tapper-Jones L, Aldred MJ, Walker DM, Hayes TM. Candidal infections and populations of Candida albicans in mouths of diabetics. J Clin Pathol 1981;34:706-711.

28. Atkinson JC, Fox PC. Salivary gland dysfunction. Clin Geriatr Med 1992;8:499-511.

29. Hernandez YL, Daniels TE. Oral candidiasis in Sjögren's syndrome: prevalence, clinical correlations, and treatment. Oral Surg Oral Med Oral Pathol 1989;68:324-329.

30. Epstein JB, Freilich MM, Nhu DL. Risk factors for oropharyngeal candidiasis in patients who receive radiation therapy for malignant conditions of the head and neck. Oral Surg Oral Med Oral Pathol 1993;76:169-174.

31. Ueta E, Osaki T, Yoneda K, Yamamoto T. Prevalence of diabetes mellitus in odontogenic infections and oral candidiasis: An analysis of neutrophil suppression. J Oral Pathol Med 1993;22:168-174.

32. Aly FZ, Blackwell CC, Mackenzie DA et al. Factors influencing oral carriage of yeast among individuals with diabetes mellitus. Epidemiol Infect 1992;109:507-518.

33. Hill LVH, Tan MH, Pereira LH, Embil JA. Association of oral candidiasis with diabetic control. J Clin Pathol 1989;42:502-505.

34. Lamey P-J, Darwazeh AMG, Muirhead J et al. Chronic hyperplastic candidosis and secretor status. J Oral Pathol Med 1990;20:64-67.

35. MacGregor IDM. Effects of smoking on oral ecology: A review of the literature. Clin Prev Dent 1989;11:3-7.

36. Challacombe SJ. Immunological aspects of oral candidiasis. Oral Surg Oral Med Oral Pathol 1994;78:202-210.

37. Dreizen S, McCredie KB, Bodey GP, Keating MJ. Quantitative analysis of the oral complications of antileukemia chemotherapy. Oral Surg Oral Med Oral Pathol 1986;62:650-653.

38. Wahlin YB. Salivary secretion rate, yeast cells, and oral candidiasis in patients with acute leukemia. Oral Surg Oral Med Oral Pathol 1991;71:689695.

39. Dreizen S, Bodey GP, Valdivieso M. Chemotherapy-associated oral infections in adults with solid rumors. Oral Surg Oral Med Oral Pathol 1983;55:113-120.

40. lacopino AM, Wathen WF. Oral candidal infection and denture stomatitis: A comprehensive review. J Am Dent Assoc 1992;123:46-51.

41. Porter SR, Scully C. Orofacial manifestations in primary immunodeficiencies involving IgA deficiency. J Oral Pathol Med 1993;22:117-119.

42. Jenkins WMM, MacFarlane TW, Ferguson MM, Mason DK. Nutritional deficiency in oral candidosis. Int J Oral Surg 1977;6:204-210.

43. Samaranayake L.P, MacFarlane TW, Lamey P-J, Ferguson MM. A comparison of oral rinse and imprint sampling techniques for the detection of yeast, coliform and Staphylococcus aureus carriage in the oral cavity. J Oral Pathol 1986;15:386-388.

44. Santarpia RP III, Pollock JJ, Renner RP, Spiechowicz E. An in vivo replica method for the site-specific detection of Candida albicans on the denture surface of denture stomatitis patients: Correlation with clinical disease. J Prosthet Dent 1990;63:437-443.

45. Epstein JB, Pearsall NN, Truelove EL. Quantitative relationships between Candida albicans in saliva and the clinical status of human subjects. J Clin Microbiol 1980;12:475-476.

46. Theilade J, Budtz-Jørgensen E. Electron microscopic study of denture plaque. J Biol Buccale 1980;8:287-297.

47. Ödman PA. The effectiveness of an enzyme-containing denture cleanser. Quintessence Int 1992;23:187-190.

48. Lal K, Santarpia RP, Pollack JJ, Renner RP. Assessment of antimicrobial treatment of denture stomatitis using an in vivo replica model system: Therapeutic efficacy of an oral rinse. J Prosthet Dent 1992;67:72-77.

49. Banting DW, Greenhorn, PA, McMinn JG. Effectiveness of a topical antifungal regimen for the treatment of oral candidiasis in older, chronically ill, institutionalized adults. J Can Dent Assoc 1995;61:199-205.

50. Nakamoto K, Tamamoto $M$, Hamada $T$. Evaluation of denture cleansers with and without enzymes against Candida albicans. J Prosthet Dent 1991;66:792-795

51. Lambert JP, Kolstad R. Effect of a benzoic acid-detergent germicide on denture-borne Candida albicans. J Prosthet Dent 1986;55:699-700.

52. Gwinnett AJ, Caputo $\mathrm{L}$. The effectiveness of ultrasonic denture cleaning: $A$ scanning electron microscopic study. J Prosthet Dent 1983;50:20-25.

53. Stafford GD, Arendorf GD, Huggett R. The effect of overnight drying and water immersion on candidal colonization and properties of complete dentures. J Dent 1986;14:52-56.

54. Martin M, Farrelly P, Hardy P. An investigation of the efficacy of nystatin for the treatment of chronic atrophic candidosis (denture sore mouth). $\mathrm{Br}$ Dent J 1986;160:201-204.

55. Athar $\mathrm{M}$, Winner $\mathrm{H}$. The development of resistance by Candida species to polyene antibiotics in vitro. J Med Microbio 1971;4:505-517.

56. Fan-Havard P, Capano D, Smith SM et al. Development of resistance in Candida isolates from patients receiving prolonged antifungal therapy. Antimicrob Agents Chemother 1991;35:2302-2305.

57. Kirkpatrick $\mathrm{CH}$, Alling DW. Treatment of chronic oral candidiasis with clotrimazole troches: A controlled clinical trial. N Engl J Med 1978;299:12011203.

58. Blatchford NR. Treatment of oral candidosis with itraconazole: A review. J Am Acad Dermatol 1990;23:565-567.

59. Budtz-Jorgensen E, Homstrup P, Krogh P. Fluconazole in the treatment of Candida-associated denture stomatitis. Antimicrob Agents Chemother 1988;32:1859-1863.

60. Kulak Y, Arikan A, Delibalta N. Comparison of three different treatment methods for generalized denture stomatitis. J Prosthet Dent 1994;72:283288.

61. Barkvoll P, Attramadal A. Effect of nystatin and chlorhexidine digluconate on Candida albicans. Oral Surg Oral Med Oral Pathol 1989;67:279-281.

62. Epstein JB, Vickars L, Spinelli J, Reece D. Efficacy of chlorhexidine and nystatin rinses in prevention of oral complications in leukemia and bone marrow transplantation. Oral Surg Oral Med Oral Pathol 1992;73:682-689.

63. Ruskin JD, Wood RP, Bailey MR et al. Comparative trial of oral clotrimazole and nystatin for oropharyngeal candidiasis prophylaxis in orthotopic liver transplant patients. Oral Surg Oral Med Oral Pathol 1992;74:567-571.

64. Ferretti GA, Ash RC, Brown AT et al. Chlorhexidine for prophylaxis against oral infections and associated complications in patients receiving bone marrow transplants. J Am Dent Assoc 1987;114:461-467.

65. Samonis G, Rolston K, Karl C et al. Prophylaxis of oropharyngeal candidiasis with fluconazole. Rev Infect Dis 1990;12(suppl 3):S369-373.

66. Scher EA, Ritchie GM, Flowers DJ. Antimycotic denture adhesive in treatment of denture stomatitis. J Prosthet Dent 1978;40:622-627.

67. Truhlar MR, Shay K, Sohnle P. Use of a new assay technique for quantification of antifungal activity of nystatin incorporated in denture liners. J Prosthet Dent 1994;71:517-524.

68. Santarpia RP, Pollock JJ, Renner RP, Gwinnett AJ. In vivo antifungal efficacy of salivary histidine-rich polypeptides: Preliminary findings in a denture stomatitis model system. J Prosthet Dent 1991;66:693-699.

69. Encarnacion M, Chin I. Salivary nystatin concentrations after administration of an osmotic controlled release tablet and pastille. Eur J Clin Pharmacol 1994:46:533-535.

70. Parvinen T, Kokko J, Yli-Urpo A. Miconazole lacquer compared with gel in treatment of denture stomatitis. Scand J Dent Res 1994;102:361-366. 